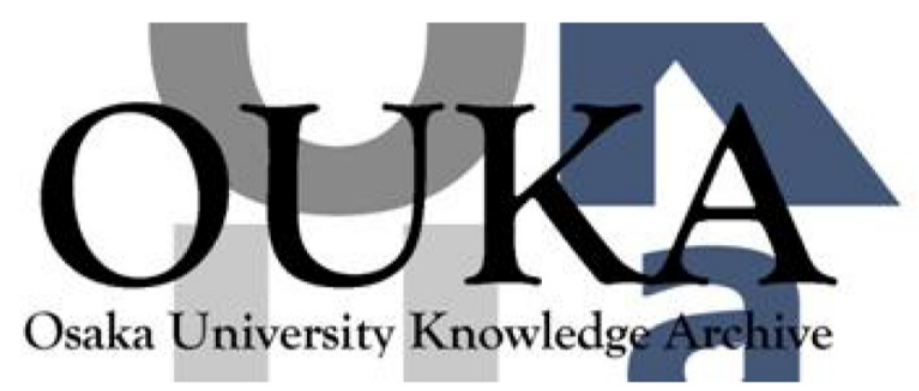

\begin{tabular}{|c|l|}
\hline Title & $\begin{array}{l}\text { Annealing effect on acoustic property of Fe/Pt } \\
\text { superlattice studied by picosecond ultrasound }\end{array}$ \\
\hline Author(s) & $\begin{array}{l}\text { Nakamura, Nobutomo; Uranishi, Atsuyoshi; } \\
\text { Wakita, Mamoru et al. }\end{array}$ \\
\hline Citation & $\begin{array}{l}\text { Japanese Journal of Applied Physics. 49 } \\
\text { p.07HB04 }\end{array}$ \\
\hline Issue Date & $2010-07-20$ \\
\hline oaire:version & AM \\
\hline URL & https://hdl. handle. net/11094/84481 \\
\hline rights & \\
\hline Note & \\
\hline
\end{tabular}

Osaka University Knowledge Archive : OUKA

https://ir. Library. osaka-u. ac. jp/

Osaka University 


\title{
Annealing Effect on Acoustic Property of Fe/Pt Superlattice Studied by Picosecond Ultrasound
}

\author{
Nobutomo Nakamura*, Atsuyoshi Uranishi, Mamoru Wakita, Hirotsugu Ogi, and \\ Masahiko HiRAO
}

Graduate School of Engineering Science, Osaka University, 1-3 Machikaneyama, Toyonaka, Osaka 560-8531, Japan

$\mathrm{Fe} / \mathrm{Pt}$ superlattices deposited on glass substrates were annealed after deposition, and the relationships among annealing temperature, out-of-plane longitudinal-wave elastic constant, and the attenuation of a $\mathrm{GHz}$ longitudinal acoustic pulse propagating in the superlattices were studied using picosecond ultrasound. Picosecond ultrasound generates and detects $\mathrm{GHz}$ longitudinal acoustic pulses using ultrashort pulse lights, and is capable of evaluating the acoustic property of a superlattice thinner than 100 $\mathrm{nm}$ without touching the specimen. The elastic constant of an as-deposited superlattice was smaller than those of bulk Fe and Pt; it increased as annealing temperature increased. The attenuation also increased as annealing temperature increased. The frequency dependence of the attenuation indicated the formation of FePt alloy at interfaces at $300{ }^{\circ} \mathrm{C}$. Thus, we confirmed that the investigation of acoustic properties using picosecond ultrasound can be a powerful tool for evaluating the structural evolution in superlattices.

\footnotetext{
*E-mail address: nobutomo@me.es.osaka-u.ac.jp
} 


\section{Introduction}

Superlattices have been attracting much attention because of their anomalous physical properties such as perpendicular magnetic anisotropy ${ }^{1)}$ and giant magnetic resistance ${ }^{2}$. Among these properties, elastic property has been widely investigated since the report on the supermodulus effect ${ }^{3)}$. Superlattices are multilayer films consisting of layers of angstrom-order thickness. The local stiffness at the interface between different materials is considered to seriously affect their macroscopic elastic constants. Moreover, interfacial defects, alloy phases, and stress due to lattice misfits are possible factors that may change local stiffness ${ }^{4-9}$. Among them, interfacial defects such as dislocations and nanoscale defects usually degrade physical properties. Annealing treatment is one of the possible techniques for decreasing the volume fraction of interfacial defects and improving physical properties, and thus its effect on elastic constant has been studied ${ }^{10,11)}$.

The annealing effect on $\mathrm{Fe} / \mathrm{Pt}$ superlattices has been an important research topic, because its elucidation can lead to the fabrication of a chemically ordered face-centered tetragonal (fct) alloy, $L 1_{0} \mathrm{FePt}$, by low-temperature annealing. $L 1_{0}$ FePt consists of alternate stacks of monoatomic layers of $\mathrm{Fe}$ and $\mathrm{Pt}$ in the [001] direction, and shows a large uniaxial magnetic anisotropy in the [001] direction. An alloy film is typically fabricated by depositing constituent materials simultaneously from different material sources, so-called codeposition, followed by annealing. In the case of $L 1_{0} \mathrm{FePt}$, codeposition requires an annealing temperature above $700{ }^{\circ} \mathrm{C}^{12)}$. However, the annealing of $\mathrm{Fe} / \mathrm{Pt}$ superlattices decreases annealing temperature to approximately $500{ }^{\circ} \mathrm{C}^{13,14)}$. $L 1_{0} \mathrm{FePt}$ can be a candidate high-density magnetic recording medium, and the lowering of its annealing temperature is an important task for its industrial use. Therefore, intensive studies have been performed on relationships among the deposition condition, magnetic property, and microstructure of FePt thin films.

Considering that the elastic constant is sensitive to the microstructure, measurement of elastic constant can be a powerful tool for elucidating structural evolution by annealing in superlattices. In addition, we focus on the attenuation of ultrahigh-frequency acoustic waves, because they also show a relationship with the microstructure. In this study, we measure elastic constant and attenuation in annealed $\mathrm{Fe} / \mathrm{Pt}$ superlattices using picosecond ultrasound coupled with X-ray reflectivity measurement, and investigate the relationships among microstructure, elastic constant, and attenuation.

\section{Experimental Procedure}

$\mathrm{Fe} / \mathrm{Pt}$ superlattices were fabricated by depositing Fe and Pt alternately on borosilicate glass substrates using RF magnetron sputtering. The thickness of each layer was $2 \mathrm{~nm}$, and the number of layers was 15: $\left[\mathrm{Pt}\left(d_{\mathrm{Pt}}=2 \mathrm{~nm}\right) / \mathrm{Fe}\left(d_{\mathrm{Fe}}=2 \mathrm{~nm}\right)\right]_{15} /$ glass. The background pressure was less than $8.0 \times 10^{-6} \mathrm{~Pa}$, and the Ar pressure was $1.3 \mathrm{~Pa}$. After depositing $\mathrm{Fe} / \mathrm{Pt}$ superlattices at room temperature, they were annealed in vacuum for $1 \mathrm{~h}$. The annealing 
temperature was 300 or $400{ }^{\circ} \mathrm{C}$. After the annealing, they were cooled down to $270{ }^{\circ} \mathrm{C}$ at a rate of $4{ }^{\circ} \mathrm{C} / \mathrm{min}$, and then naturally cooled to ambient temperature.

The microstructure was investigated by X-ray reflectivity and X-ray diffraction analyses. When X-rays irradiate a film surface at a small incident angle, two kinds of X-ray interference occur: one is the interference between X-rays reflected on the film surface and that reflected at the interface with the substrate, and the other is the interference of X-rays reflected at interfaces between Fe and Pt layers. These interferences cause some peaks in low-angle regions of the X-ray reflectivity spectrum. By measuring the peak angles, the total film thickness $d$ and the bilayer thickness $d_{b}=d_{\mathrm{Fe}}+d_{\mathrm{Fe}}$ are determined ${ }^{15,16)}$. Structural evolution by annealing was investigated by X-ray diffraction analysis.

Among independent elastic constants, the longitudinal-wave elastic constant in the film thickness direction, $C_{\perp}$, was focused on and determined using picosecond ultrasound. When the $x_{3}$-axis is aligned perpendicular to the film surface, and the $x_{1}$ - and $x_{2}$-axes are in plane, $C_{33}$ in the elastic constant matrix corresponds to $C_{\perp}$. $C_{\perp}$ is sensitive to thin inclusions aligned parallel to the film surface, and the measurement is suitable for studying structural evolution at interfaces. Picosecond ultrasound is a technique for generating and detecting the $\mathrm{GHz}$ longitudinal acoustic pulse propagating in the film-thickness direction using ultrashort-pulse lights, which is capable of measuring the round-trip time $\Delta t$ of the acoustic phonon repeating reflections between the film surface and the film-substrate interface ${ }^{17-21)} . C_{\perp}$ is determined from $\Delta t, d$, and the mass density $\rho$, through $C_{\perp}=\rho(2 d / \Delta t)^{2} . \rho$ was calculated from the mass densities of Fe and Pt as $\rho=\left(\rho_{\mathrm{Fe}}+\rho_{\mathrm{Pt}}\right) / 2$. We used a pulse-laser beam of $800 \mathrm{~nm}$ wavelength at $20 \mathrm{~mW}$ and that of $400 \mathrm{~nm}$ wavelength at $5 \mathrm{~mW}$ for generation and detection, respectively. Details of our optics appear elsewhere ${ }^{22}$.

\section{Results and Discussion}

Figure 1 shows the X-ray reflectivity spectra, in which two kinds of peaks appear: a broad peak at approximately $2 \theta=2.5^{\circ}$ and small peaks with small periodicities at $2 \theta=1.3-2.5^{\circ}$. The broad peak originates from the interference between X-rays reflected at the interfaces between the Fe and Pt layers, and the peak angle is closely related to $d_{b}$. The appearance of this peak confirms the periodic structure. The smaller-period peaks originate from the interference between X-rays reflected at the film surface and superlattice-substrate interface. Total film thickness determines this periodicity. In all superlattices, small peaks were observed, which confirms that the film surface was only slightly roughened by the annealing, and that $d$ was determined from the peak angles.

In Fig. 2, X-ray diffraction spectra show significant structural changes in the $\mathrm{Fe} / \mathrm{Pt}$ superlattice. Typical diffraction peaks regarding Fe(110), Pt(111), and $\mathrm{FePt}(111)$ are shown by dashed lines. In as-deposited and $300^{\circ} \mathrm{C}$ superlattices, a peak appeared at $2 \theta=49.5^{\circ}$, which is the fundamental peak, being associated with the multilayer structure. At approximately 
$47^{\circ}$, another peak appeared. Considering the multilayer structure of the FePt superlattice, this peak should be the satellite peak. However, the diffraction angle is also close to that of $\mathrm{Pt}(111)$, and an overlap between the satellite and diffraction peaks of $\mathrm{Pt}(111)$ can occur. Annealing at $300{ }^{\circ} \mathrm{C}$ increased the intensity, indicating that crystallinity was improved; the curing of interfacial defects is a possible reason for this. Fundamental peaks appeared between the peak angles of the $\mathrm{Fe}(110)$ and $\mathrm{Pt}(111)$ planes, which indicates that the $\langle 110\rangle$ direction of $\mathrm{Fe}$ and the $\langle 111\rangle$ direction of $\mathrm{Pt}$ are aligned in the film thickness direction. At $400{ }^{\circ} \mathrm{C}$, the fundamental and satellite peaks disappeared, and a peak appeared at $48.5^{\circ}$. The diffraction angle is close to that of $\mathrm{FePt}(111)$, i.e., $48.3^{\circ}$, rather than to that of $\mathrm{Fe}_{3} \mathrm{Pt}(111), 49.0^{\circ}$. Therefore, we consider that $L 1_{0} \mathrm{FePt}$ was formed at the interfaces. In this superlattice, no other diffraction peaks were observable except for this peak. However, X-ray reflectivity measurement confirmed its periodic structure, and the superlattice should consist of Fe and Pt layers; the $L 1_{0} \mathrm{FePt}$ alloy phase formed at Fe-Pt interfaces. The observed structural transition from a multilayer to an alloy induced by annealing is similar to that reported in the literature ${ }^{13)}$, but the transition temperature is higher in this study. The differences in the number of stacking layers and in thicknesses of $\mathrm{Fe}$ and Pt layers are possible reasons for this.

Figure 3 shows a typical reflectivity change measured by picosecond ultrasound. The horizontal axis shows the delay time of the probe beam, and the vertical axis shows the change ratio of reflectivity. The reflectivity change shows a train of echo signals, and $\Delta t$ is determined from the slope of the relationship between delay time and the index number of echo signals, $m$.

Figure 4 shows the $C_{\perp}$ of the $\mathrm{Fe} / \mathrm{Pt}$ superlattice, which is compared with the elastic constants of bulk Fe and Pt. The bulk materials' elastic constants, $C_{\perp}^{\mathrm{Fe}(110)}$ and $C_{\perp}^{\mathrm{Pt}(111)}$, were calculated from the elastic constants of the monocrystals $\mathrm{Fe}^{23)}$ and $\mathrm{Pt}^{24)}$ by coordinate transformation, assuming that the $\mathrm{Fe}(110)$ and $\mathrm{Pt}(111)$ planes are parallel to the film surface, referring to the X-ray diffraction spectra.

The $C_{\perp}$ of the as-deposited Fe/Pt superlattice was significantly smaller than both $C_{\perp}^{\mathrm{Fe}(110)}$ and $C_{\perp}^{\mathrm{Pt}(111)}$. However, it increased as annealing temperature increased, and reached a value between $C_{\perp}^{\mathrm{Fe}(110)}$ and $C_{\perp}^{\mathrm{Pt}(111)}$. Interfacial dislocations can soften the superlattice ${ }^{7)}$. Considering that such defects were cured by annealing, the compliant as-deposited superlattice and the increase in $C_{\perp}$ induced by annealing can be consistently explained. At $400{ }^{\circ} \mathrm{C}$, the formation of an alloy phase could also contribute to the increase, but its contribution to the elastic constant cannot be simply explained by this result.

From the reflectivity changes observed by picosecond ultrasound, echo signals were extracted, and fast Fourier transform (FFT) spectra were calculated to determine the frequency components in the acoustic pulse. The FFT spectra of the first and third echoes $(m=1$ and 3) are shown in Fig. 5. The center frequency of the first echo was about $50 \mathrm{GHz}$ in all su- 
perlattices, but at $m=3$, the center frequency was comparable to or smaller than $50 \mathrm{GHz}$. This result indicates that the attenuation of higher-frequency components is higher. Figure 6 shows the attenuation coefficient $\alpha$ of several frequency components, which was deduced by fitting the exponential function $\exp (-\alpha t)$ to FFT spectra of $m=1,2,3$, and 4 . $\alpha$ increased as frequency increased, and the behavior differed depending on annealing temperature. It should be noted that $\alpha$ contains energy loss due to the transmission of acoustic waves into the substrate. Because the degree of leakage is independent of frequency, its contribution to $\alpha$ is negligible in the following discussion concerning the frequency dependence of $\alpha$.

In polycrystalline bulk materials, when the wavelength is much larger than the grain size, the following equation has been successfully used for explaining the frequency dependence of $\alpha^{25,26)}$ :

$$
\alpha=a_{1} f+a_{4} f^{4}
$$

where $f$ is the frequency, $a_{1}$ the elastic hysteresis coefficient, and $a_{4}$ the Rayleigh scattering coefficient. This equation indicates that attenuation contains a hysteresis term proportional to frequency and a fourth-power scattering term. The former indicates absorption loss due to, for example, dislocation. Near the dislocation-line resonance, a $f^{2}$ dependence is observed. However, because of the narrow frequency range, we used a linear frequency dependence. The frequency dependence of attenuation related to scattering varies depending on the wavelength of the acoustic wave and the size of the inclusion. In superlattices, a locally formed alloy inclusion can scatter acoustic waves. The wavelength of acoustic phonons in the $\mathrm{Fe} / \mathrm{Pt}$ superlattice is about $90 \mathrm{~nm}$ at $50 \mathrm{GHz}$, which is much larger than the thickness of each layer. Therefore, we assume Rayleigh scattering by inclusions. To determine the contributions of the absorption and scattering terms, $\alpha$ was fitted using $\alpha=A_{0} f^{n}+A_{1}$, where $A_{0}$ and $A_{1}$ are constants, and the contribution of each factor is evaluated by deducing $n$. When $n$ is close to 1 , the attenuation is attributed to the hysteresis term. As $n$ increases, the contribution of scattering term increases. In Fig. 6 , the $\alpha$ of the as-deposited superlattice apparently shows a linear dependence on frequency $(n=1.8)$. However, at $300^{\circ} \mathrm{C}, \alpha$ increases rather than show a linear-dependence with the increase in frequency $(n=2.3)$, which indicates that the scattering term in eq. (1) contributes to the attenuation. This is attributed to the formation of the alloy phase at interfaces. In X-ray diffraction spectra, no formation of FePt alloy was observed, but the annealing caused diffusion, forming local alloy phase inclusions at the Fe-Pt interface. Considering the scattering induced by the alloy inclusion, the larger $n$ at $300^{\circ} \mathrm{C}$ is explained. At $400^{\circ} \mathrm{C}$, an $L 1_{0} \mathrm{FePt}$ alloy was formed at the Fe-Pt interface, and the Fe/Pt superlattice consisted of Fe, Pt, and FePt layers, which could decrease the contribution of scattering $(n=0.93)$. Alternately, attenuation by the absorption term was increased by the formation of the alloy layer. 


\section{Conclusions}

We observed that the $C_{\perp}$ of the $\mathrm{Fe} / \mathrm{Pt}$ superlattice increased as annealing temperature increased, and the contribution of scattering on the attenuation varied depending on annealing temperature. From the X-ray diffraction analysis, the formation of an FePt alloy phase by annealing was observed, but no details on the interfacial structure could be deduced. However, the annealing temperature dependences of elastic constant and attenuation provide valuable information concerning the interfacial structure, which is difficult to obtain by X-ray diffraction analysis. We consider that the use of picosecond ultrasound in combination with typical structural analysis is a powerful tool for elucidating the microstructures of superlattices. 


\section{References}

1) P. F. Carcia, A. D. Meinhaldt and A. Suna: Appl. Phys. Lett. 47 (1985) 178.

2) P. Grünberg, R. Schreiber, Y. Pang, M. B. Brodsky, and H. Sowers: Phys. Rev. Lett. 57 (1986) 2442.

3) W. M. C. Yang, T. Tsakalakos, and J. E. Hilliard: J. Appl. Phys. 48 (1977) 876.

4) H. Huang and F. Spaepen: Acta Mater. 48 (2000) 3261.

5) B. M. Clemens and G. L. Eesley: Phys. Rev. Lett. 61 (1988) 2356.

6) N. Nakamura, H. Ogi, T. Ono, M. Hirao, and M. Nishiyama: Jpn. J. Appl. Phys. 44 (2005) 4427.

7) N. Nakamura, H. Ogi, T. Yasui, M. Fujii, and M. Hirao: Phys. Rev. Lett. 99 (2007) 035502.

8) H. Tanei, N. Nakamura, Y. Kake, H. Ogi, K. Kusakabe, and M. Hirao: Jpn. J. Appl. Phys. 47 (2008) 3847.

9) H. Ogi, T. Shagawa, N. Nakamura, and M. Hirao: Appl. Phys. Express 2 (2009) 105001.

10) G. Cariotti, D. Fioretto, G. Socino, L. Verdini, and V. Pelosin: J. Appl. Phys. 73 (1993) 3028.

11) S. Lee, G. I. Stegeman, J. Kim, C. M. Falco, V. Askarpour, and M. H. Manghnani: Solid State Commun. 94 (1995) 691.

12) Y. K. Takahashi, K. Hono, T. Shima, and K. Takanashi: J. Magn. Magn. Mater. 267 (2003) 248.

13) Y. Endo, N. Kikkuchi, O. Kitakami, and Y. Shimada: J. Appl. Phys. 89 (2001) 7065.

14) F. Casoli, F. Albertini, L. Pareti, S. Fabbrici, L. Nasi, C. Bocchi, and R. Ciprian: IEEE Trans. Magn. 41 (2005) 3223.

15) H. Kiessig: Ann. Phys. (Leipzig) 10 (1931) 769 [in German].

16) L. G. Parratt: Phys. Rev. 95 (1954) 359.

17) C. Thomsen, J. Strait, Z. Vardeny, H. J. Maris, J. Tauc, and J. J. Hauser: Phys. Rev. Lett. 53 (1984) 989.

18) C. Thomsen, H. T. Grahn, H. J. Maris, and J. Tauc: Phys. Rev. B 34 (1986) 4129.

19) D. H. Hurley and O. B. Wright: Opt. Lett. 24 (1999) 1305.

20) O. Matsuda, O. B. Wright, D. H. Hurley, V. E. Gusev, and K. Shimizu: Phys. Rev. Lett. 93 (2004) 095501.

21) N. Nakamura, H. Ogi, H. Nitta, H. Tanei, M. Fujii, T. Yasui, and M. Hirao: Jpn. J. Appl. Phys. 45 (2006) 4580.

22) H. Ogi, M. Fujii, N. Nakamura, T. Yasui, and M. Hirao: Phys. Rev. Lett. 98 (2007) 195503.

23) C. A. Rotter and C. S. Smith: J. Phys. Chem. Solids 27 (1966) 267.

24) R. E. Macfarlane, J. A. Rayne, and C. K. Jones: Phys. Lett. 18 (1965) 91.

25) W. P. Mason and H. J. Mcskimin: J. Appl. Phys. 19 (1948) 940.

26) E. P. Papadakis and E. L. Reed: J. Appl. Phys. 32 (1961) 682. 


\section{Figure Captions}

Fig. 1 X-ray reflectivity spectra of $[\mathrm{Pt}(2 \mathrm{~nm}) / \mathrm{Fe}(2 \mathrm{~nm})]_{15} /$ glass measured by CoK $\alpha$ radiation. The vertical axis is on a logarithmic scale.

Fig. 2 X-ray diffraction spectra of $[\mathrm{Pt}(2 \mathrm{~nm}) / \mathrm{Fe}(2 \mathrm{~nm})]_{15} /$ glass annealed at several temperatures measured using $\operatorname{CoK} \alpha$ radiation. The vertical axis is on a linear scale.

Fig. 3 Reflectivity change of the as-deposited Fe/Pt superlattice of $59.3 \mathrm{~nm} . m$ is the index number of pulse-echo signals. The inset shows the relationship between $m$ and delay time, and is approximated by a linear function. The round-trip time of the longitudinal acoustic pulse between the superlattice surface and the superlattice-substrate interface is determined from the slope of the linear function.

Fig. 4 Out-of-plane elastic constant of $\mathrm{Fe} / \mathrm{Pt}$ superlattice annealed at several temperatures. Dashed lines show the longitudinal elastic constants in the $\langle 111\rangle$ direction of $\mathrm{Pt}$ and in the $\langle 110\rangle$ direction of Fe.

Fig. 5 Fast Fourier transform spectra of first and third echo signals $(m=1$ and 3$)$ deduced from measured reflectivity changes.

Fig. 6 Attenuation coefficient at several frequencies of echo signals, which is fitted by $A_{0} f^{n}+$ $A_{1}$. 


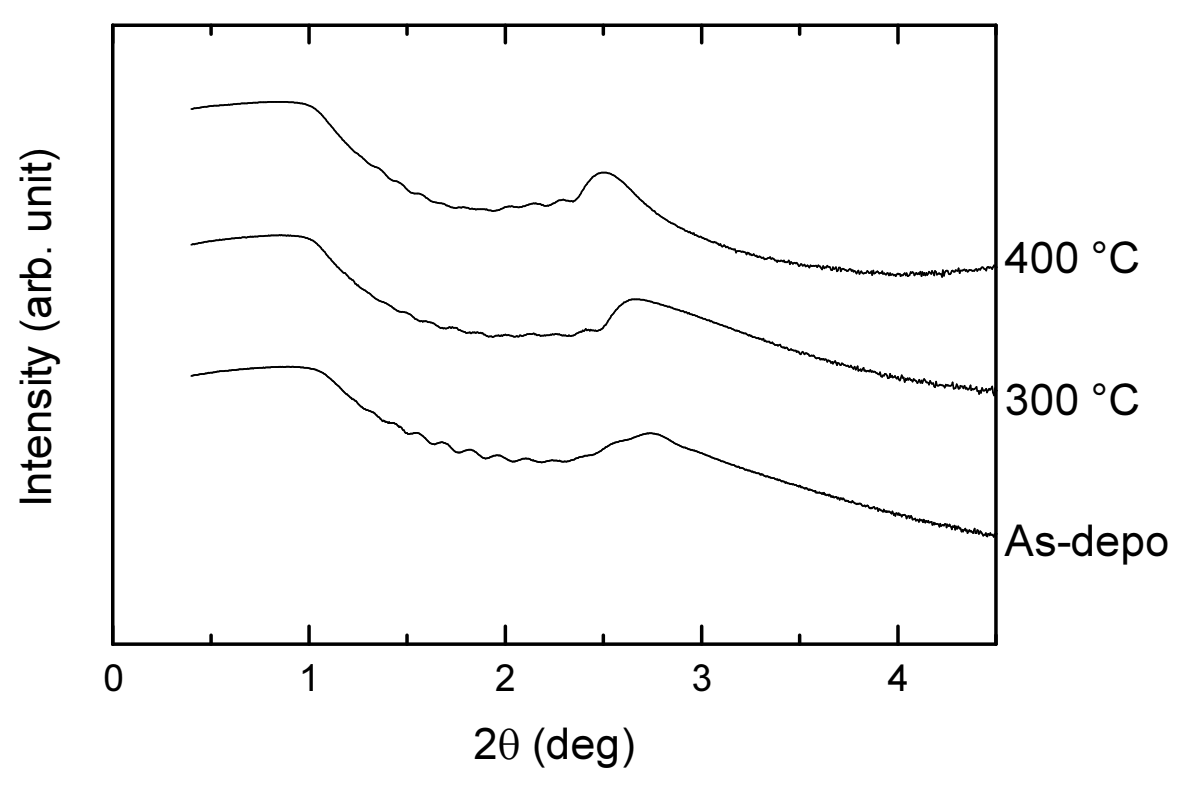

Fig. 1. 


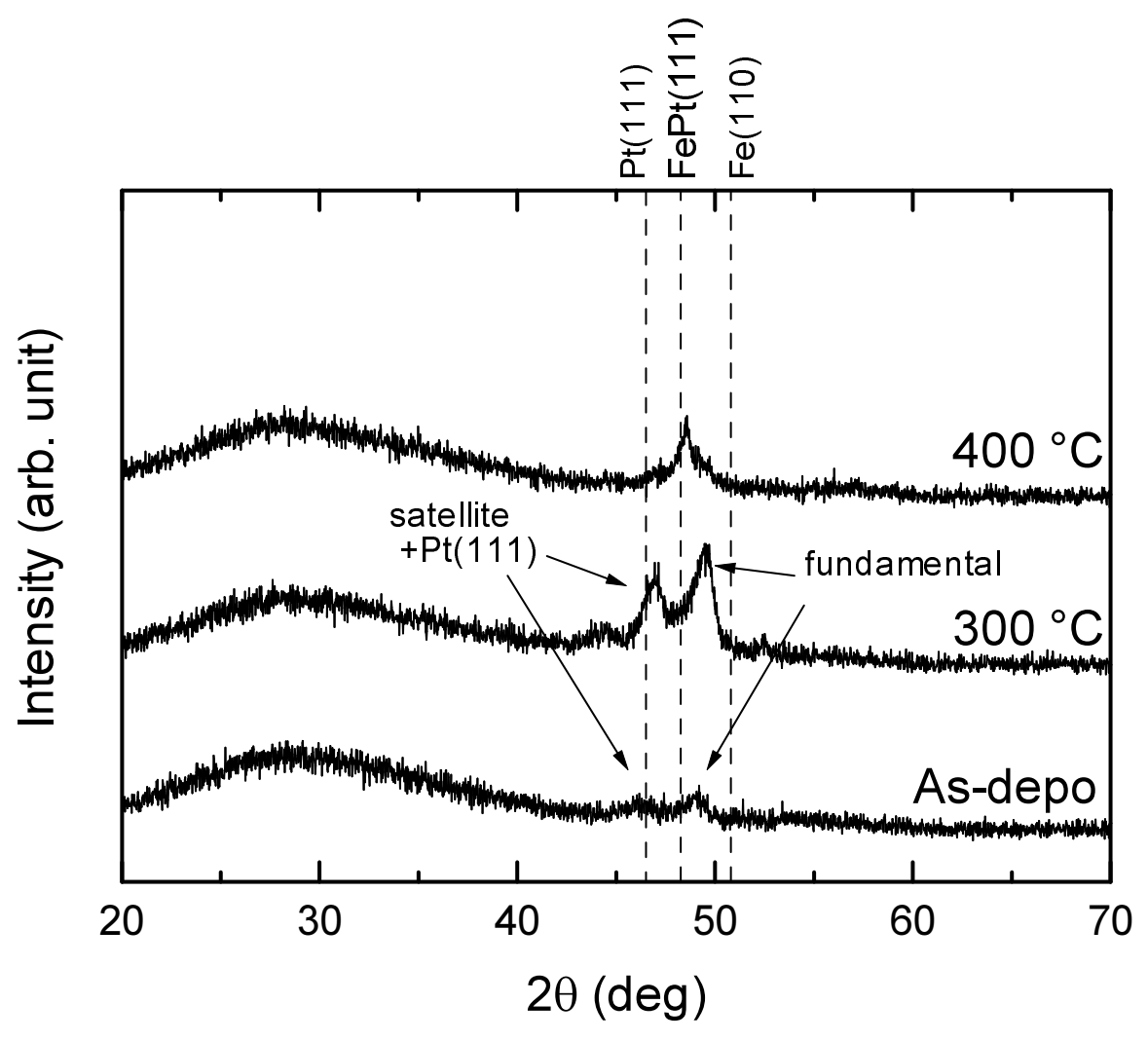

Fig. 2. 


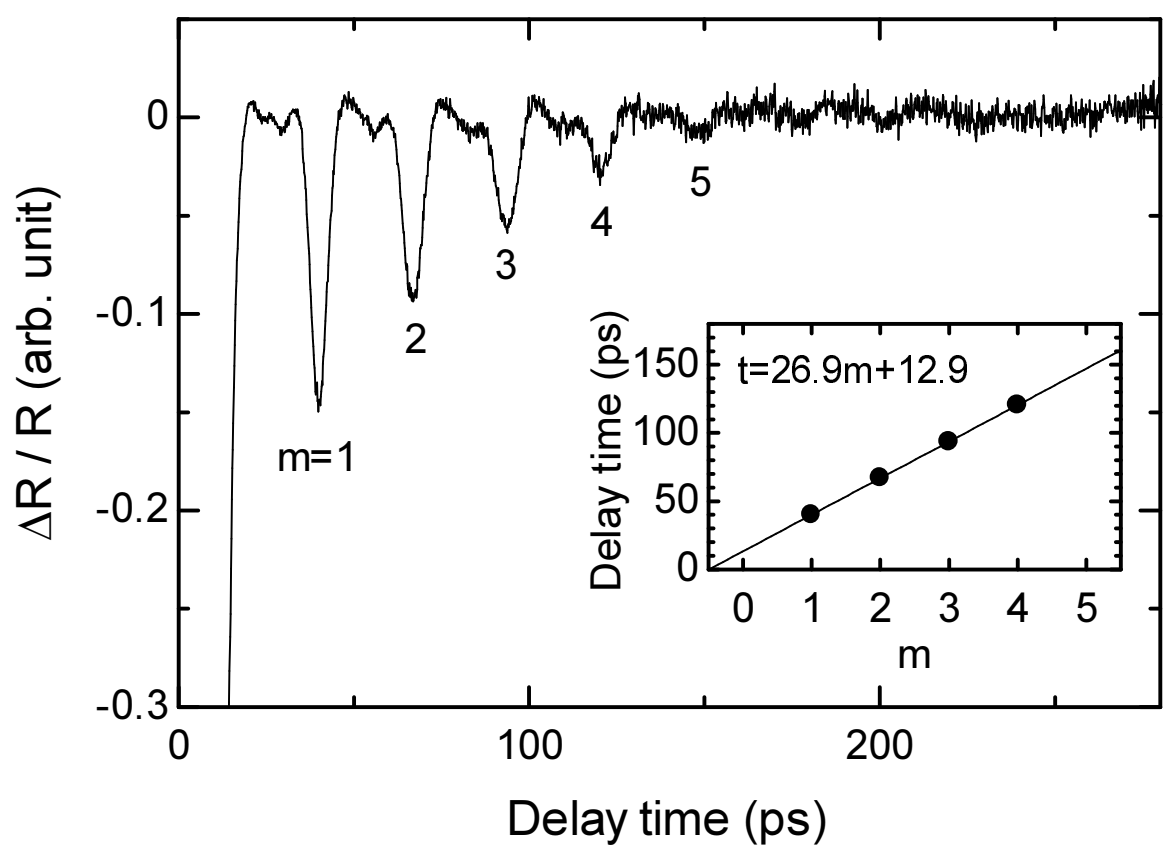

Fig. 3. 


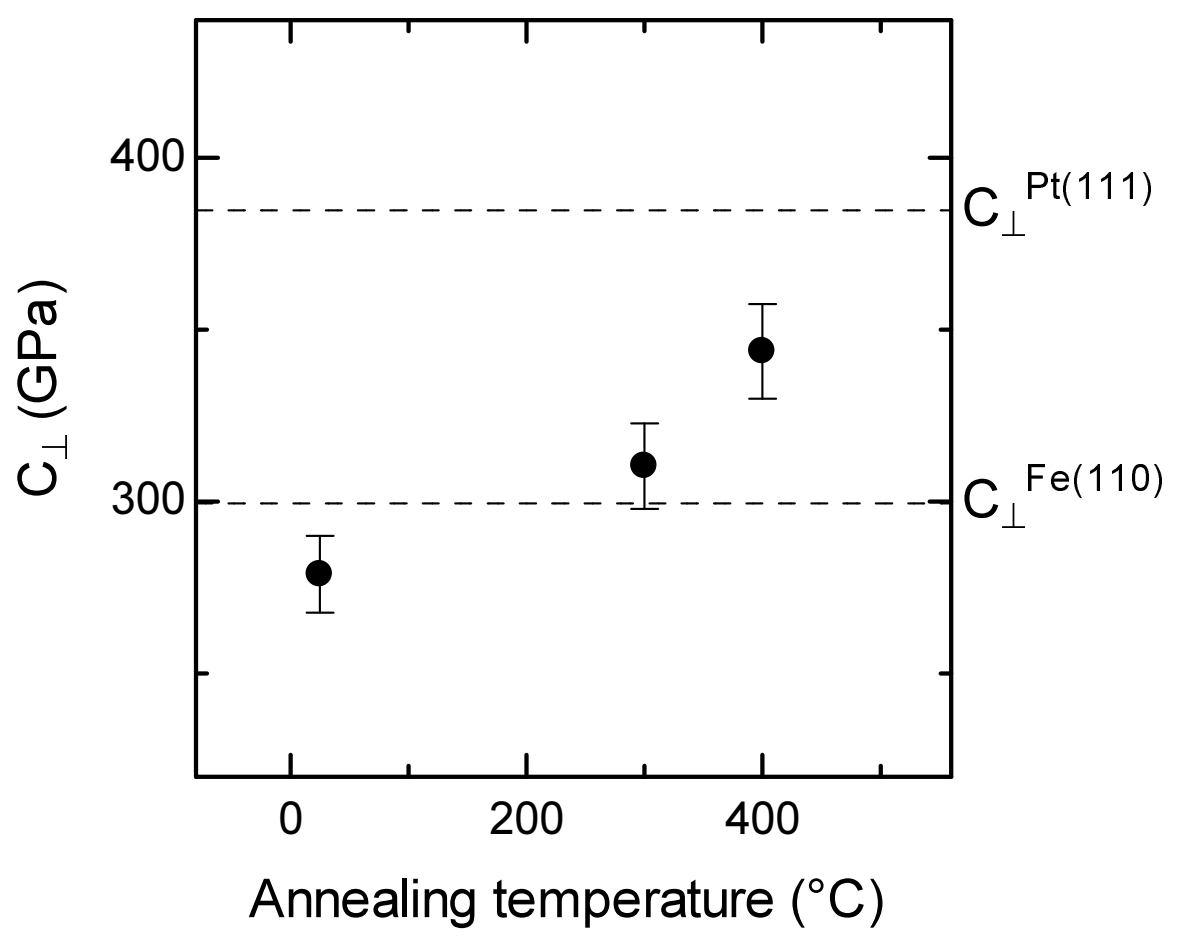

Fig. 4. 


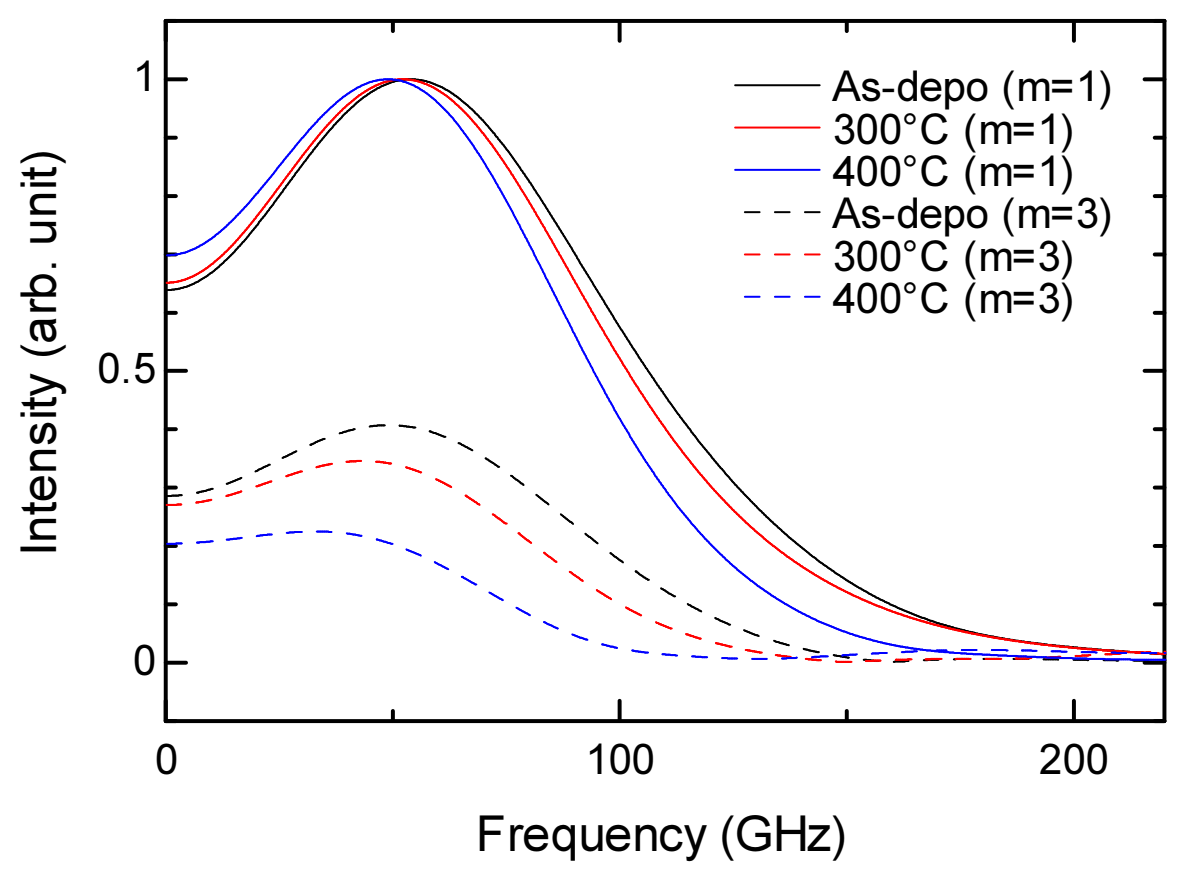

Fig. 5. 


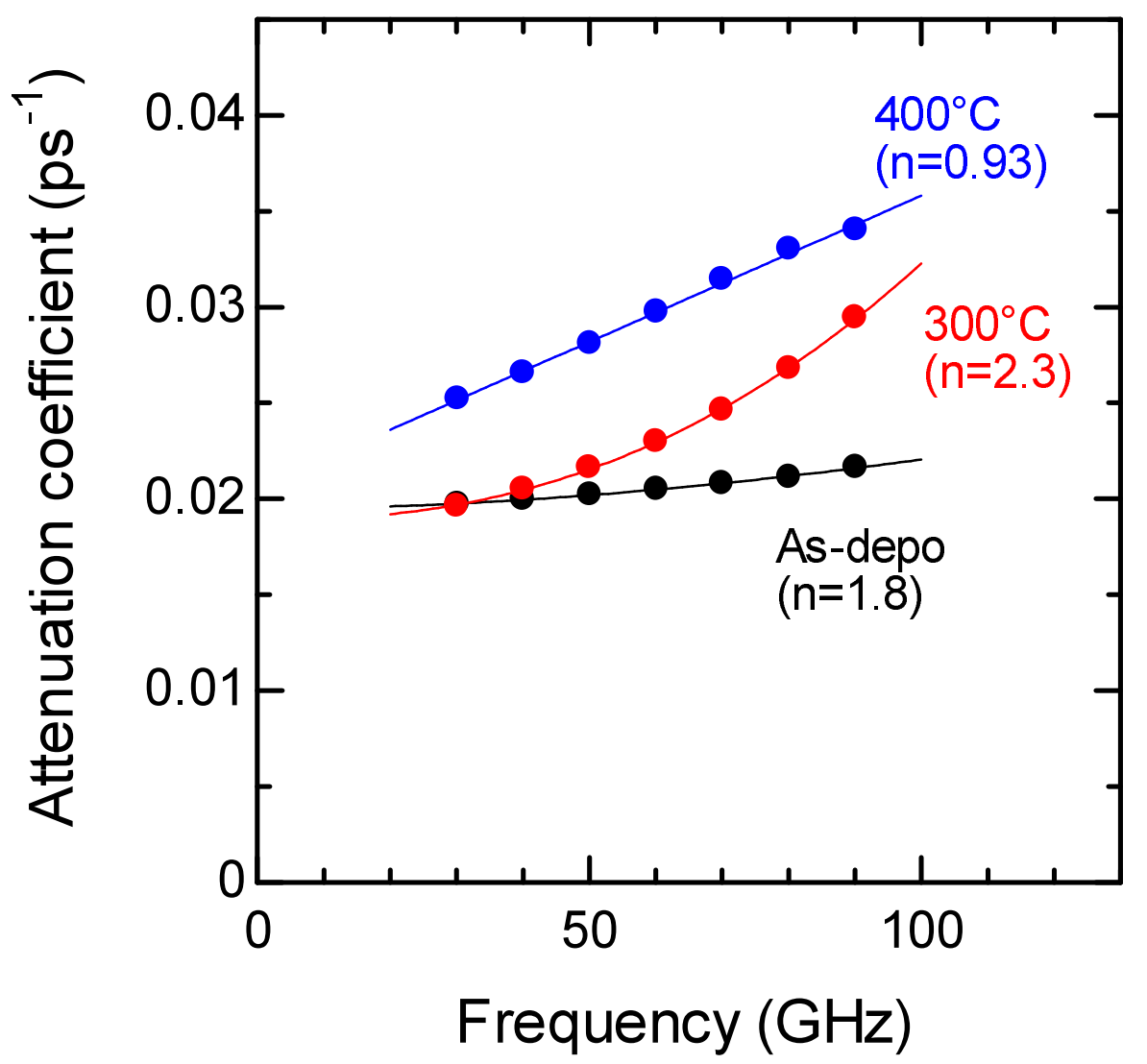

Fig. 6 . 